Research Paper

\title{
Comparison of the Prognostic Value of Systemic Inflammation Response Markers in Small Cell Lung Cancer Patients
}

\author{
Ting Zhou ${ }^{1,2,3^{*}}$, Yuanyang Zhao ${ }^{1,2,3^{*}}$, Shen Zhao ${ }^{1,2,3^{*}}$, Yunpeng Yang1,2,3, Yan Huang1,2,3, Xue Hou 1,2,3, \\ Hongyun Zhao ${ }^{1,2,3}$, Li Zhang $1,2,3 凶$ \\ 1. Department of Medical Oncology, Sun Yat-Sen University Cancer Center, Guangzhou 510060, China; \\ 2. State Key Laboratory of Oncology in South China, Guangzhou 510060, China; \\ 3. Collaborative innovation Center for Cancer Medicine, Guangzhou 510060, China, \\ *These authors contributed equally to this work and share the first authorship. \\ $\triangle$ Corresponding author: Dr. Li Zhang, Department of Medical Oncology, Sun Yat-Sen University Cancer Center, 651 Dongfeng East Road, Guangzhou, 510060, \\ China; Tel: +862087343822; Fax: +862087343392; Email: zhangli6@mail.sysu.edu.cn
}

(C) Ivyspring International Publisher. This is an open access article distributed under the terms of the Creative Commons Attribution (CC BY-NC) license (https://creativecommons.org/licenses/by-nc/4.0/). See http://ivyspring.com/terms for full terms and conditions.

Received: 2018.08.18; Accepted: 2019.01.12; Published: 2019.04.03

\begin{abstract}
Increasing evidences support that systemic inflammation-based prognostic scores, modified Glasgow Prognostic Score (mGPS), C-reactive Protein/Albumin (CRP/ALB), Albumin/Globulin (AGR), Prognostic Nutritional Index (PNI) and Advanced Lung cancer Inflammation index (ALI), are key determinants of patients' outcome in solid tumors. However, in small cell lung cancer (SCLC), there have been no direct comparisons of them. Thus, the aim of this study was to compare the prognostic value of these markers in SCLC, and select a most appropriative one. The patients with confirmed SCLC were screened between 2006 and 2011, and inflammation-based prognostic factors (mGPS, CRP/ALB, AGR, PNI, ALI) were examined. Kaplan-Meier and Cox regression analysis were performed to assess these inflammation-based prognostic scores associated with overall survival (OS). Subsequently, we compared the prognostic value of these inflammation-based prognostic scores using the area under the curve (AUC). In 451 patients, on univariate analysis, mGPS ( $P<0.001)$, CRP/ALB $(P<0.001), A G R(P<0.001), P N I$ $(P<0.001)$ and $A L I(P<0.001)$ were the strongest predictors of $O S$. Further multivariate analysis confirmed mGPS $(P<0.001)$, CRP/ALB $(P=0.007), \operatorname{AGR~}(P=0.034)$ and PNI $(P=0.026)$ as independent markers associated with OS. Further subgroup analysis revealed CRP/ALB was able to predict outcome in both limited $(P=0.005)$ and extensive disease $(P=0.013)$. The CRP/ALB had higher AUC values compared with other inflammation-based prognostic socres (0.566). The CRP/ALB was characterized as best, in comparison to other systemic inflammation-based prognostic scores, for its predictive power of SCLC patients' survival, and had the potential to be hierarchical factor in future clinical trials.
\end{abstract}

Key words: small cell lung cancer, systemic inflammation-based prognostic scores, C-reactive protein, albumin, prognosis

\section{Background}

With identification of 4292,000 new cases, and 2814,000 cancer related deaths in the year 2015, lung cancer remains the most common malignant tumor type and the leading cause of cancer deaths in China [1]. Based on different biological and morphological characteristics, lung cancer is categorized into two major types; Small cell lung cancer (SCLC) and non-small cell lung cancer (NSCLC). Among these,
SCLC accounts for approximately $15 \%$ of all lung cancers [2], and is typically characterized by its highly aggressive nature and early tendency to distant metastasis [3]. The Veteran Affairs Lung Study Group (VALSG) classification further categorized SCLC patients into limited stage (LS) and extensive stage (ES) [4]. Unfortunately, due to the development of frequent chemotherapeutic resistance, most LS-SCLC 
patients, and nearly all ES-SCLC patients, suffer from recurrence and progression, and subsequently result in their poor prognosis $[5,6]$. Moreover, due to existence of heterogeneity and variable clinical characteristics, the SCLC prognosis can vary among different patients. Therefore, it is critical and necessary to find an appropriate individual stratified variable, which can help to guide better anti-tumor and palliative treatment options in SCLC patients.

It is widely recognized that variations in patient prognosis in cancer are not solely determined by tumor characteristics, but patient-response factors also play an important role [7]. Over the years, it has increasingly been evident that cancer-associated inflammation, especially the host systemic inflammation response, show close association with disease development and progression, along with patient survival in cancers $[8,9]$. Concurrently, the levels of acute-phase proteins, like C-reactive protein (CRP) and albumin (ALB) are assessed in the plasma to evaluate the degree of systemic inflammation [10]. In addition, multiple studies have also demonstrated about the prognostic power of these factors in variety of cancers, independent of disease stage, performance status and clinical characteristics [11]. Besides, some cellular components of the inflammatory response, including lymphocytes and neutrophils, have also been reported to depict predictive value in the outcome of patients with malignant tumors $[12,13]$.

Interestingly, it was realized early on that combining these factors into some kind of systemic inflammatory based scores, can greatly help to develop some independent prognostic value. Among them, modified Glasgow Prognostic Score (mGPS), which consist of CRP and ALB concentrations, was regarded as a prominent prognostic factor in most cancers [14]. Also, the prognostic nutritional index (PNI), calculated based on albumin level and total lymphocyte count, was also effective at predicting overall survival [15]. Similarly, neutrophil-to-lymphocyte ratio (NLR) was also noticed to be beneficial in predicting the survival in solid tumors [16].

In recent years, several studies have indicated that host response to systemic inflammation is still an important factor in determining the clinical outcomes in SCLC patients $[17,18]$. Indeed, our previous studies have also concluded that such systemic inflammation based prognostic scores, including mGPS, CRP/ALB and PNI, were useful in predicting cancer-specific survival in SCLC patients [19-21]. Because of the objectivity, reliability and accessibility of these systemic inflammation based prognostic scores, it is obvious that their use can be adapted in clinical settings, and can help to stratify SCLC patients. However, to our knowledge, no direct comparison of the longitudinal measurements of these systemic inflammation based prognostic scores has been undertaken in SCLC.

Therefore, the present study was planned to compare the prognostic value of selected systemic inflammation response markers in SCLC patients, and also identify a most appropriate systemic inflammation based prognostic score.

\section{Methods}

\section{Patients}

Patients with histologically confirmed SCLC, between January 2006 and December 2011, at Sun Yat-sen University Cancer Center, were included in our study. Other eligible selection criteria were as follows: (a) patients displaying Eastern Cooperative Oncology Group performance status (PS) of 2 or less; (b) are older than 18 years; (c) had available pre-treatment laboratory data, consisting of CRP and ALB levels, along with lymphocyte and neutrophil counts; (d) displayed normal liver function; and (e) had sufficient clinical information. However, the patients were excluded if they; (a) had other types of cancers; (b) had liver disease; (c) had clinical evidence of infection; and (e) had autoimmune disease. The written informed consent was obtained from each patient before the start of study.

\section{Biomarkers and other variables}

CRP, total protein, albumin, neutrophil count, and lymphocyte count, considered as biomarkers of systemic inflammation response were measured from the patient's blood samples, collected before the initial treatment. The detailed scoring criteria were summarized in Supplementary Table S1.

The mGPS was calculated as follows [22]: $\mathrm{CRP} \leq 10 \mathrm{mg} / \mathrm{L}=0, \mathrm{CRP}>10 \mathrm{mg} / \mathrm{L}=1, \mathrm{CRP}>10 \mathrm{mg} / \mathrm{L}$ and albumin $<35 \mathrm{~g} / \mathrm{L}=2$. The CRP/ALB ratio was calculated by dividing the CRP level by albumin level [23]. The AGR was defined as follows [24]:

AGR= albumin (g/L) / (total protein- albumin) (g/L)

The PNI was calculated as follows [25]:

$\mathrm{PNI}=10 \times$ albumin $(\mathrm{g} / \mathrm{L})+0.005 \times$ lymphocyte count $\left(10^{9} / \mathrm{L}\right)$

The ALI was calculated with the following formula [26]:

$$
\begin{gathered}
\text { ALI= BMI }\left(\mathrm{Kg} / \mathrm{m}^{2}\right) \times \text { albumin }(\mathrm{g} / \mathrm{L}) /(\text { neutrophil } \\
\text { count/ lymphocyte count })
\end{gathered}
$$

The information about other baseline clinical characteristics, consisting of demographic information, performance status (PS), smoking status, body mass index (BMI), cancer stage and therapeutic 
information, was also collected. The smokers were defined as patients who had more than 100 cigarettes. The SCLC staging was determined according to VALG staging system.

\section{Treatment and patient follow-up}

The most frequent chemotherapy administered to the patients consisted of platinum plus etoposide, on day 1 through 3 , for 4 or 6 cycles, after every 3 weeks. Some patients even received thorax radiotherapy (TRT) along with chemotherapy. Furthermore, several patients subsequently underwent prophylactic cranial irradiation (PCI).

All the enrolled patients were carefully assessed for therapy response after every two cycles of treatment, using dynamic computed tomography (CT) scan. After completion of the anti-tumor treatment, patients received CT scan every 2 months thereafter, or at the time when any symptoms of possible progression were observed. Tumor response evaluation was carried out according to Response Evaluation Criteria in Solid Tumors (RECIST) version 1.1. Patient overall survival (OS) was defined as the period from the time of pathological diagnosis to the date of death for any cause or last follow-up. Patients who didn't die at the date of cutoff were censored, and the last follow-up date was December 2017.

\section{Statistical analysis}

The primary outcome of our study was overall survival. Pearson correlation, Chi-square test, and Fisher exact test were used to compare continuous and categorical variables. Kaplan-Meier method was used to estimate the probability of survival, and logrank test was performed to assess survival difference. The predictive power of potential prognostic variables, including sex, PS, cancer stage, mGPS, CRP/ALB, AGR, PNI, as well as ALI, were assessed using Cox proportional hazards model. The optimal cut-off value of CRP/ALB, AGR, PNI and ALI were determined using an $\mathrm{R}$ software-engineered, web-based system designed by Budczies J et al. (http://molpath. charite.de/cutoff/) [27]. The P value of less than 0.05 represented statistically significant difference. All of the statistical tests were two-tailed and all statistical analyses were performed using SPSS statistical software package (version 21.0, IBM, Armonk, NY).

\section{Results}

\section{Patients Characteristics}

A total of 591 SCLC diagnosed patients were reviewed at our center, and among them 46 patients did not have any baseline data about patient height or weight, while 94 patients had no information about pre-treatment CRP levels. Therefore, only 451 eligible
SCLC patients were included for further analysis in our study (Figure 1).

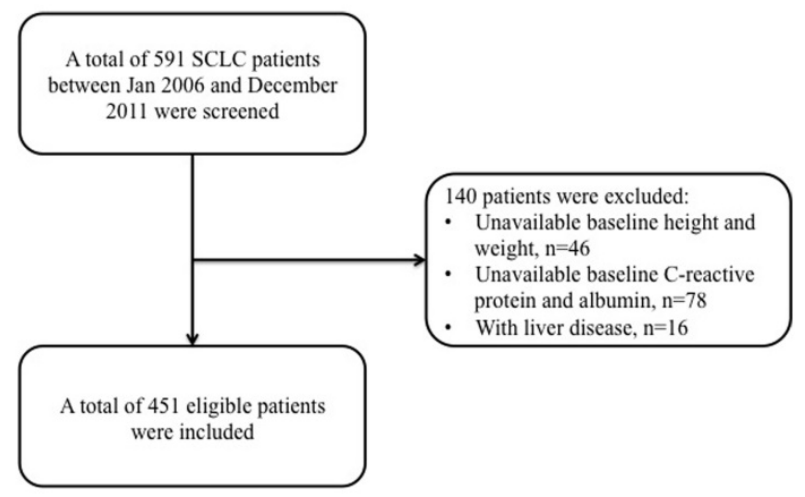

Figure 1. Flow chart of patients' enrollment.

The baseline characteristics of all these patients have been shown in Table 1. Overall, the median age of patients was 60 years (range: 19-82 years), and majority of them were male $(86.3 \%, 389$ of 451 patients), smokers $(82.0 \%, 370$ of 451 patients), and displayed limited stage disease $(55.4 \%, 250$ of 451 patients) as characterized by VALG criteria. Eastern Cooperative Oncology Group PS was 0 in 257 patients $(57.0 \%), 1$ in 158 patients $(35.0 \%)$, and 2 in 36 patients $(18.0 \%)$. In addition, $85.4 \%(\mathrm{n}=385)$ patients received etoposide-based chemotherapy regimen, while 75 (29.9\%) patients had TRT, and another 64 (25.5\%) patients received PCI after chemotherapy.

\section{Correlation between patient survival and clinicopathological characteristics}

During the follow-up period, 306 (67.8\%) of the total patients died, and the observed median OS of enrolled patients was 15.6 months (range: 0.03-92.03 months). The relationship between clinicopathological characteristics of SCLC patients and their survival has been summarized in Table 1 . The univariate survival analysis showed following parameters as significantly associated with overall survival; PS $(P<0.001)$, LDH level $(P<0.001)$, tumor stage $(P<0.001)$, and TRT $(P=0.003)$. Further, multivariate analysis only confirmed PS $(P<0.001)$, LDH level $(P=0.002)$ and tumor stage $(P<0.001)$ as independent prognostic factors related with cancer survival.

\section{Correlation between patient survival and inflammation biomarkers}

In addition, we assessed the prognostic power of CRP, ALB, Neutrophil (NE), and lymphocyte (LY), with an intency to identify the association between patient survival and systemic inflammation biomarkers. Based on the biostatistical tool Cutoff Finder, the optimal cutoff values of CRP, ALB, NE, LY were 5.8, 
36.0, 5.0, and 1.8 for OS, respectively. The univariate survival analysis demonstrated elevated CRP $(P<0.001)$ and NE $(P=0.010)$ levels, along with reduced ALB $(P<0.001)$ and LY $(P=0.023)$ levels, to be significantly associated with poor cancer-specific survival (Supplemental Figure 1). However, multivariate analysis only validated CRP [hazard ratio (HR) 1.32, 95\% CI 1.03-1.70, $P=0.032$ ] and ALB (HR 1.77, 95\% (CI) 1.19-2.64, $P=0.005$ ) levels to be independently associated with cancer-specific survival.

\section{Correlation between patient survival and systemic inflammation-based prognostic scores}

As independent prognostic values of CRP and ALB have been shown above, through both univariate and multivariate analyses, we next tried to explore the potential predictive power of systemic inflammationbased prognostic scores calculated based on CRP and/or ALB levels.

mGPS

A total of $273(60.5 \%)$ SCLC patients had mGPS of 0 , while $162(35.9 \%)$ patients had mGPS of 1 . Only $16(3.5 \%)$ patients displayed mGPS of 2 at baseline (Table 2). Importantly, the patients with mGPS of 2 displayed 2.2 months of OS, and this OS period was significantly less in comparison to patients with mGPS of 0 and 1 , with OS of 19.4 and 15.9 months, respectively $(P<0.001)$ (Figure $2 \mathrm{~A})$. Interestingly, multivariate analysis revealed an independent association between mGPS (HR 5.53, 95\% CI 3.17-9.66, $P<0.001$ ) and OS in SCLC patients (Table 2).

\section{CRP/ALB}

The observed optimal cutoff point of CRP/ALB for OS was 0.44 (range: $0.00-10.40$ ). Based on this, the patients were divided into two groups: Group 1

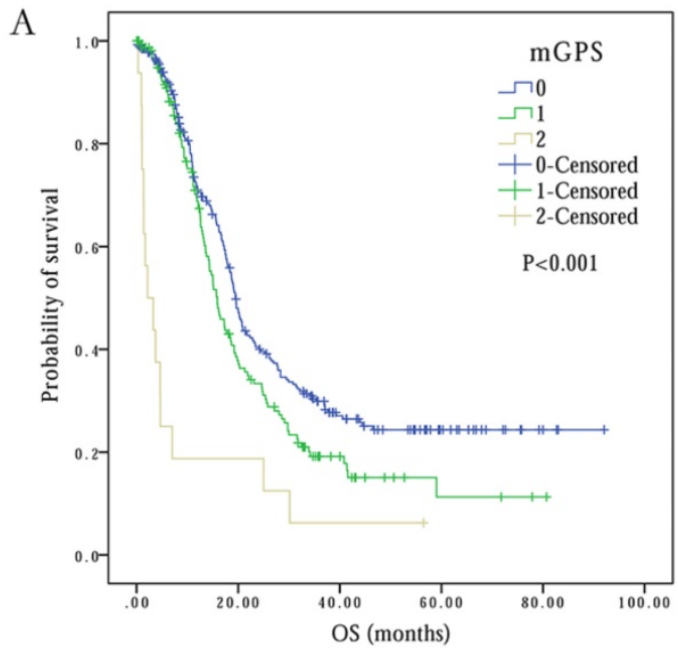

consisted of 319 (70.7\%) patients with CRP/ALB value of $\leq 0.44$, while group 2 with CRP/ALB value of $>0.44$ had $132(29.3 \%)$ patients (Table 2). The univariate survival analysis displayed that elevated CRP/ALB ratio correlated with lower OS time (13.6 months at CRP/ALB> 0.44 vs. 19.6 months at $\mathrm{CRP} / \mathrm{ALB} \leq 0.44) \quad(P<0.001)$ (Figure 2B). Multivariate survival analysis further confirmed that patients with elevated CRP/ALB ratio of $>0.44$, had 1.41 times higher risk of death (HR 1.41, 95\% CI 1.10-1.81, $P=0.007$ ) (Table 2).

AGR

The optimal cutoff level of AGR for patient OS was observed to be 1.25 (range: 0.16-4.94). Based on this cutoff value, $93(20.6 \%)$ patients were in the low AGR group, while remaining 358 (79.4\%) patients were in high AGR group (Table 2). Univariate analysis showed that overall patients survival in high AGR group was much longer than in low AGR group (19.6 months vs. 15.6 months, $P<0.001$ ) (Figure 3A). Multivariate analysis showed AGR as an independent prognostic factor in SCLC (HR 1.35, 95\% CI 1.02-1.77, $P=0.034)$ (Table 2).

\section{PNI}

With PNI optimal cutoff level of 37.5 (range: 13.5-52.5) for OS, $387 \quad(85.8 \%)$ patients were categorized into high PNI group, and 64 (14.2\%) were part of low PNI group (Table 2). Based on univariate analysis, high PNI was observed to be significantly associated with better OS, in comparison to low PNI (18.9 months vs. 12.1 months, $P<0.001$ ) (Figure 3B). Multivariate analysis also established independent association of PNI with overall survival (HR 1.19, 95\% CI 1.02-1.39, $P=0.026$ ) (Table 2).

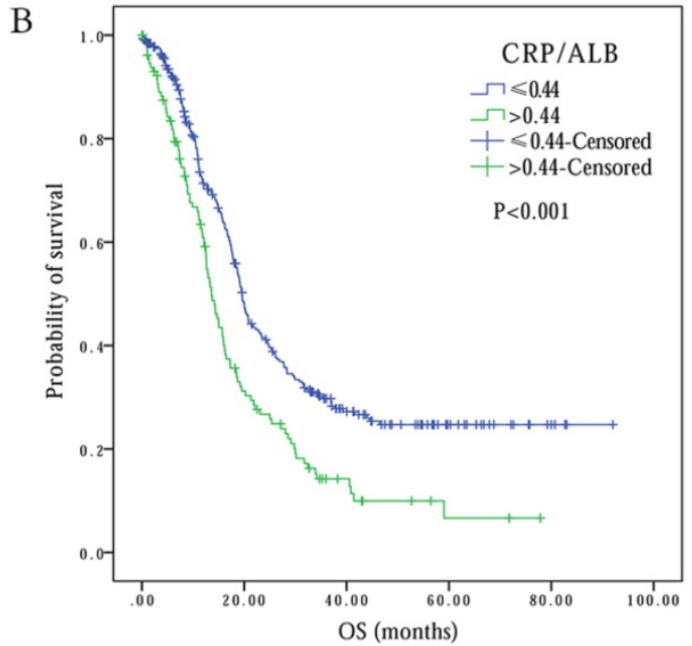

Figure 2. Kaplan-Meier survival curves comparing $S C L C$ patients with (A) $m G P S=0$ vs. $m G P S=1$ vs. $m G P S=2$, (B) $C R P / A L B \leq 0.44$ vs. $C R P / A L B>0.44$. 
Table 1. Basic characteristic of all patients and univariate survival analysis

\begin{tabular}{|c|c|c|c|c|}
\hline Variables & $\begin{array}{l}\text { Cases, } \\
N\end{array}$ & $\begin{array}{l}\text { Proportion, } \\
\%\end{array}$ & $\begin{array}{l}\text { Median OS, } \\
\text { months }(95 \% \mathrm{CI})\end{array}$ & $P$ value \\
\hline Age, years & $60(19-82)$ & & & 0.468 \\
\hline \multicolumn{5}{|l|}{ Gender } \\
\hline Male & 389 & 86.3 & $17.9(16.52-19.28)$ & 0.418 \\
\hline Female & 62 & 13.7 & $18.7(13.63-23.77)$ & \\
\hline \multicolumn{5}{|l|}{ Smoking } \\
\hline Smoker & 370 & 82.0 & $18.5(17.05-20.02)$ & 0.392 \\
\hline Never-smoker & 81 & 18.0 & 16.7(13.95-19.45) & \\
\hline \multicolumn{5}{|l|}{ Disease stage } \\
\hline Limited stage & 251 & 55.7 & $25.4(20.01-30.73)$ & $<0.001$ \\
\hline Extensive stage & 200 & 44.3 & $14.2(11.67-16.67)$ & \\
\hline \multicolumn{5}{|l|}{ PS } \\
\hline 0 & 257 & 57.0 & 19.2(17.49-20.91) & $<0.001$ \\
\hline 1 & 158 & 35.0 & 18.7(16.63-20.70) & \\
\hline 2 & 36 & 8.0 & $9.3(8.06-10.61)$ & \\
\hline \multicolumn{5}{|l|}{ TRT } \\
\hline Yes & 171 & 62.1 & 19.0(17.50-20.57) & 0.003 \\
\hline No & 280 & 37.9 & $13.6(17.50-20.57)$ & \\
\hline \multicolumn{5}{|l|}{ LDH, U/L } \\
\hline$<245$ & 282 & 62.5 & 19.8(17.15-22.45) & $<0.001$ \\
\hline$\geq 245$ & 169 & 37.5 & 13.1(10.88-15.32) & \\
\hline \multicolumn{5}{|c|}{ Chemotherapy regimen } \\
\hline Etoposide-based & 385 & 85.4 & $18.4(16.64-20.09)$ & 0.953 \\
\hline Others & 66 & 14.6 & $17.5(15.03-19.91)$ & \\
\hline \multicolumn{5}{|l|}{ PCI } \\
\hline Yes & 107 & 23.7 & 18.6(16.24-21.02) & 0.457 \\
\hline No & 344 & 76.3 & 18.0(16.31-19.76) & \\
\hline
\end{tabular}

Table 2. Results from Cox Regression Model (Adjusted for mGPS, CRPIALB, AGR, PNI, ALI)

\begin{tabular}{|c|c|c|c|c|}
\hline \multirow[t]{2}{*}{ Variables } & \multirow[t]{2}{*}{ Hazard Ratio } & \multicolumn{2}{|c|}{$95 \%$ CI } & \multirow[t]{2}{*}{$P$ value } \\
\hline & & LL & UL & \\
\hline \multicolumn{5}{|l|}{ mGPS } \\
\hline 0 & 1.00 & - & - & - \\
\hline 1 & 1.34 & 0.89 & 1.45 & 0.293 \\
\hline 2 & 5.53 & 3.17 & 9.66 & $<0.001$ \\
\hline \multicolumn{5}{|l|}{ CRP/ALB } \\
\hline$>0.44$ & 1.00 & - & - & - \\
\hline$\leq 0.44$ & 1.41 & 1.10 & 1.81 & 0.007 \\
\hline \multicolumn{5}{|l|}{ AGR } \\
\hline$\geq 1.46$ & 1.00 & - & - & - \\
\hline$<1.46$ & 1.35 & 1.02 & 1.77 & 0.034 \\
\hline \multicolumn{5}{|l|}{ PNI } \\
\hline$<37.5$ & 1.00 & - & - & - \\
\hline$\geq 37.5$ & 1.19 & 1.02 & 1.39 & 0.026 \\
\hline \multicolumn{5}{|l|}{ ALI } \\
\hline$>47.0$ & 1.00 & - & - & - \\
\hline$\leq 47.0$ & 1.11 & 0.96 & 1.28 & 0.172 \\
\hline
\end{tabular}

\section{ALI}

Based on the ALI optimal cutoff value of 47.0 (range: 4.2-544.6) for OS, the patients were divided into two groups: ALI $\leq 47.0(\mathrm{n}=341,75.6 \%)$ and ALI > $47.0(\mathrm{n}=110,24.4 \%)$ (Table 2). The univariate analysis showed negative association between low ALI and OS (25.6 months at ALI $>47.0$ vs. 16.5 months at ALI $\leq 47.0, P<0.001$ ) (Figure 3C). However, multivariate analysis did not reveal ALI as an independent predictor of overall survival in SCLC $(P=0.172)$ (Table 2).

\section{Comparison of systemic inflammation-based prognostic scores}

Finally, to compare the prognostic power of these systemic inflammation-based prognostic scores, we performed survival analysis based on cancer stage stratification. Our data showed that prognostic values of mGPS $(P<0.001)$, AGR $(P<0.001)$, PNI $(P=0.001)$ and ALI $(P<0.001)$ had significant association with patients at extension stage, but not at limited stage $(P=0.092, \quad P=0.171$ and $P=0.620$, respectively). In contrast, the association between CRP/ALB and OS was noted in both extensive $(P=0.013)$ and limited stage $(P=0.005)$ patients (Figure 4$)$. In addition, we also performed ROC curve analysis, and compared the AUC values to evaluate the differentiation ability of each systemic inflammation-based prognostic score. The CRP/ALB score displayed higher AUC value in comparison to mGPS, AGR, PNI, and ALI scores (Figure 5) $(P=0.023)$.

\section{Discussion}

The links between systemic inflammation and cancer have been explored widely for decades, because epidemiological observation have indicated that inflammatory markers are evident in the tumor microenvironment, and tumors are frequently formed at the sites of chronic inflammation [28]. Recent studies have clearly suggested about the obvious relationships between systemic inflammatory response markers and tumor patients poor outcome [29, 30]. More specifically, our study also confirmed the link of C-reactive protein and hypoalbuminemia with SCLC patient survival, but the molecular mechanism of their role is still not elucidated.

Importantly, our study established that in comparison to the cellular components of white cell counts (lymphocyte and neutrophil), the acute phase proteins (C-reactive protein and albumin) consistently have independent prognostic value. This would mean that markers like mGPS, CRP/ALB and AGR, which were calculated based on C-reactive protein and albumin levels, were definitely superior to those cellular components, including PNI and ALI, in predicting SCLC patient's survival. We believe that our study is the first, which offered detailed insight into systemic inflammation-based prognostic scores in SCLC patients.

In addition, our study also demonstrated association of systemic inflammatory response with cancer-specific survival in patients with small cell lung cancer. The association of systemic inflammation-based prognostic scores, including mGPS, AGR, $\mathrm{CRP} / \mathrm{ALB}$, and PNI with cancer-specific survival in SCLC patients, was consistent with previously published studies in other tumor types [23, 31-33]. To 
further identify a most appropriate systemic inflammation-based prognostic score, we performed a longitudinal comparison of these prognostic scores in SCLC patients, and subgroup analysis based on disease stage demonstrated that the prognostic power of CRP / ALB ratio was remarkable in both limited and extensive stage patients. However, the prognostic
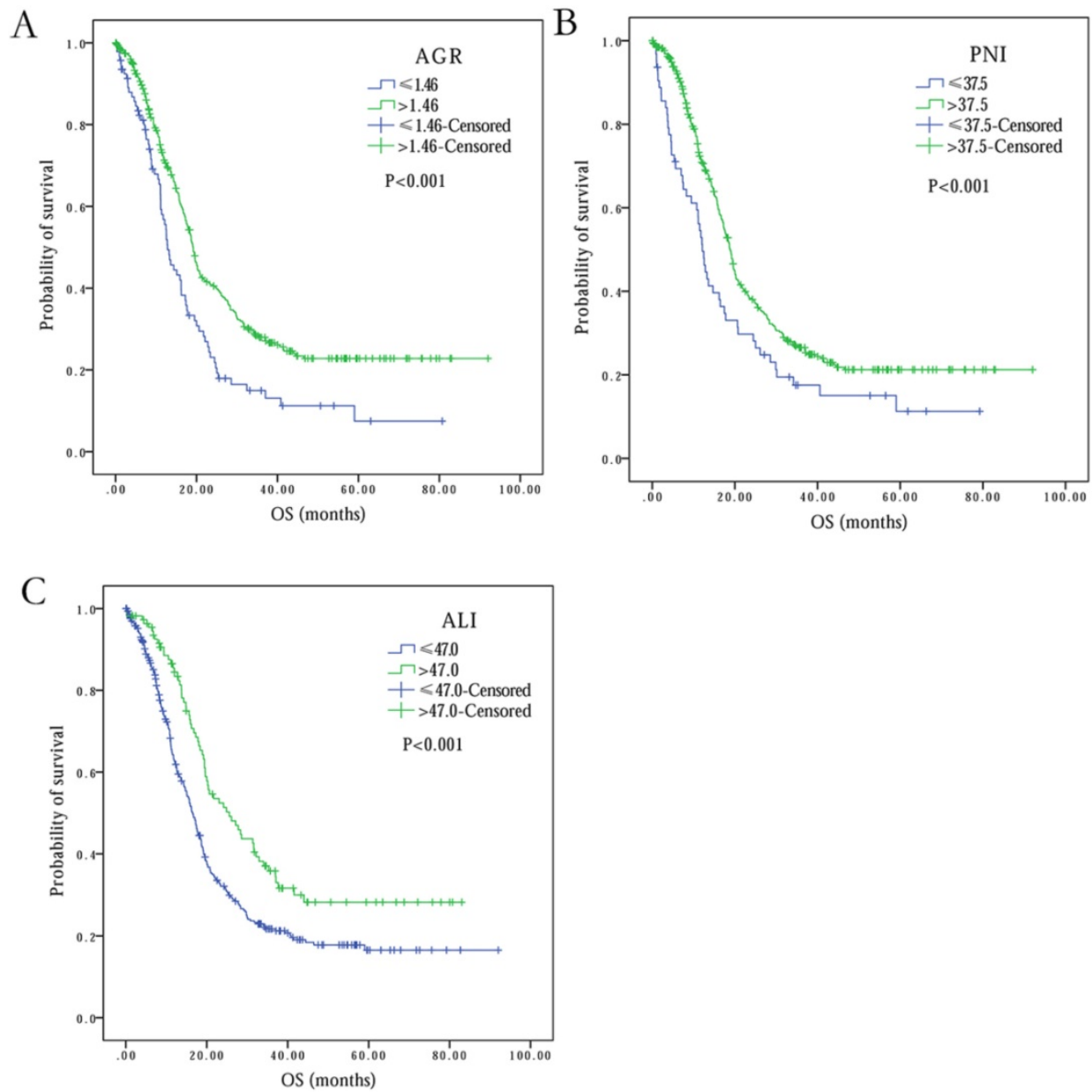

scores of other markers like, mGPS, AGR, and PNI too showed significant prognostic abilities in extensive stage patients, but did not show same prognostic power in limited stage patients. It is important to note that mGPS revealed some prognostic tendency in limited stage patients, but the results were insignificant.
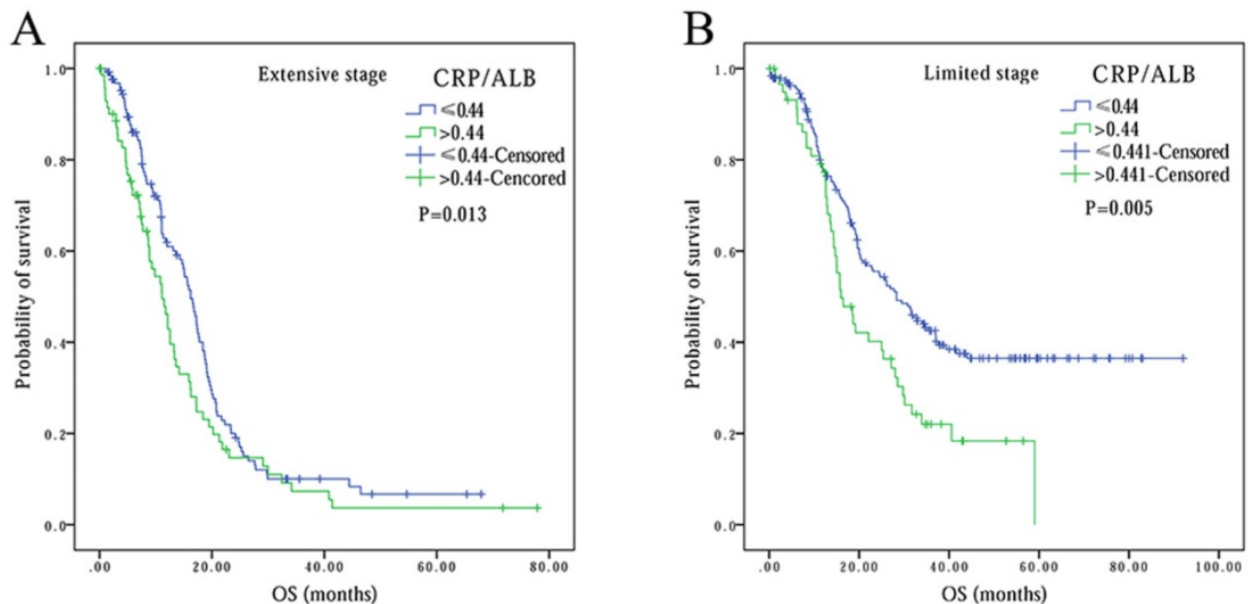

Figure 4. Kaplan-Meier survival curves comparing SCLC patients with CRP/ALB $\leq 0.44$ vs. CRP/ALB>0.44 in different stages: (A) in extensive stage, (B) in limited stage. 


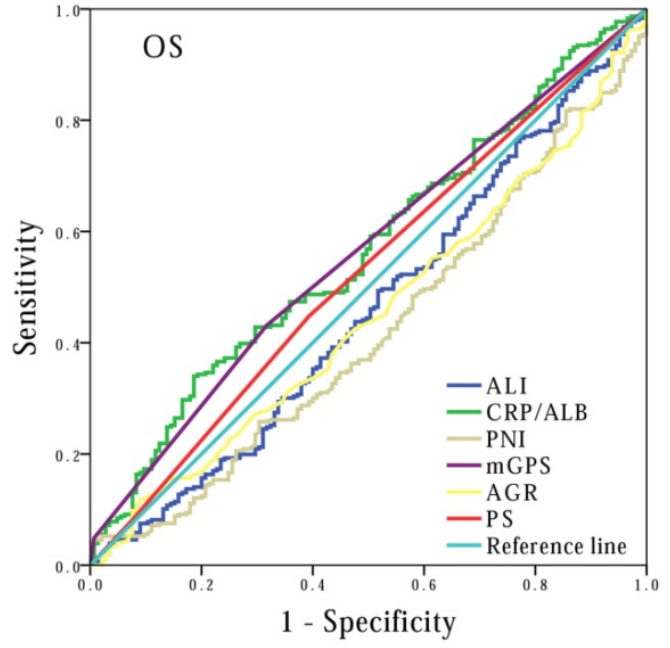

Figure 5. Comparison of the areas under the receiver operating curves for outcome prediction among the prognostic scores: CRP/ALB, mGPS, AGR, PNI and PS.

The CRP/ALB ratio was calculated based on the laboratory measurement of CRP and albumin levels. Some studies have revealed that SCLC patients have higher levels of CRP in comparison to non-malignant subjects, and show association with worse patient outcome [34, 35]. Albumin is often considered as a marker for nutritional status of patients in clinic [36], and recent studies have indicated that low baseline albumin levels not only have strong relationship with tumor progression, but also with poor overall survival in lung cancer [37, 38]. However, the biological mechanism of association between CRP, albumin and increased risk of cancer development is not clear. In this context, it is first hypothesized that CRP increase and albumin decrease is probably regulated by proinflammatory cytokines, especially interleukin (IL)-6 and IL-1. These cytokines are also crucial for neoangiogenesis and disease progression, and thus are significantly associated with the increasing risk of lung cancer development $[39,40]$. Second, an elevated CRP and reduced albumin levels correlates with increase in weight loss, fatigue and lean tissue, thereby probably resulting in poor performance status and survival in various cancer types [41,42].

Interestingly, ROC analysis suggested that AUC value of CRP/ALB was higher than mGPS, AGR, and PNI for overall survival, which implied that CRP/ALB ratio has powerful predictive value, in comparison to other inflammation-based prognostic scores. Additionally, it was also noticed that CRP/ALB ratio, based on its outstanding prognostic power, had the ability to stratify SCLC patients' outcome at diagnosis. Moreover, since ROC analysis indicated higher values for CRP/ALB ratio and mGPS than AGR and PNI, this clearly show that CRP and albumin have higher prognostic value than white cell count based prognostic scores, whether they contain albumin or not. Consistent with this observation, a previous study has also identified that CRP based prognostic scores were more powerful than other inflammation-based prognostic scores, in patients with different tumor types [43].

The study by Temel et al. has highlighted the importance of clinicians, in deciding which patients will receive subsequent therapy, and who would receive only palliative care [44]. In fact the clinicians are the one who will make decision about the quality of life and anti-tumor therapy administration in cancer patients. Thus, they importantly need an appropriate prognostic marker, which will subsequently help them to predict between the patients response and the value of anti-tumor therapy. In this context, our study clearly suggested that CRP/ALB ratio is the marker that is powerful enough to act as prognostic score in SCLC patients. The other advantage of CRP/ALB ratio as a prognostic score is economical, as it is based only on two baseline laboratory makers, which are simple and inexpensive. Thus, it can be really helpful in improving SCLC patients stratification.

Finally, our study also has some limitations, including it being a retrospective and single center study, and thus has the potential to suffer from selection bias. However, to reduce the bias, we have recruited consecutive patients. Moreover, multivariate analysis showed CRP/ALB as an independent prognostic factor, after adjustment for other clinical characteristics. Therefore, a multi-center and largescale prospective validation study would be essentially required to establish these findings independently.

Overall, our study concluded that systemic inflammation response makers are vital predictors of overall survival in SCLC patients. Especially, we observed that CRP/ALB ratio is not only an independent prognostic marker, but also seems to be most optimal score, among all other analyzed systemic inflammation-based prognostic scores, in terms of its predictive power of SCLC patient survival. In addition, CRP/ALB ratio appears to be cost-effective prognostic factor, and can be easily adapted in the clinical practice to stratify the patients for future clinical trials.

\section{Supplementary Material}

Supplementary figures and tables.

http://www.jcancer.org/v10p1685s1.pdf

\section{Acknowledgements}

This work was supported by: $\mathrm{Wu}$ Jieping Medical Foundation Project (Grant No: 08-JC-003), Innovative drug $R \& D$ center based on real-time 
high-throughput cell-based screening platform and large capacity compound library (Grant No: 2013ZX09 401003-002), National Natural Science Funds of China (Grant No: 81372502) and National High Technology Research and Development Program of China (Grant No: 2012AA02A502). All the grant supporting agencies have no roles in study design, data collection and analysis, and manuscript preparation.

\section{Competing Interests}

The authors have declared that no competing interest exists.

\section{References}

1. Chen W, Zheng R, Baade PD, et al. Cancer statistics in China, 2015. CA Cancer J Clin. 2016;66:115-32.

2. Byers LA, Rudin CM. Small cell lung cancer: where do we go from here? Cancer. 2015;121:664-72.

3. van Meerbeeck JP, Fennell DA, De Ruysscher DK. Small-cell lung cancer. Lancet. 2011:378:1741-55.

4. Micke P, Faldum A, Metz T, et al. Staging small cell lung cancer: Veterans Administration Lung Study Group versus International Association for the Study of Lung Cancer-what limits limited disease? Lung Cancer. 2002;37:271-6.

5. Lawson MH, Cummings NM, Rassl DM, et al. Two novel determinants of etoposide resistance in small cell lung cancer. Cancer Res. 2011;71:4877-87.

6. Gardner EE, Lok BH, Schneeberger VE, et al. Chemosensitive Relapse in Small Cell Lung Cancer Proceeds through an EZH2-SLFN11 Axis. Cancer Cell. 2017;31:286-99.

7. MacDonald N. Cancer cachexia and targeting chronic inflammation: a unified approach to cancer treatment and palliative/supportive care. J Support Oncol. 2007;5:157-162; discussion 164-156, 183.

8. Mantovani A. Cancer: Inflaming metastasis. Nature. 2009;457:36-7.

9. Mantovani A, Allavena P, Sica A, et al. Cancer-related inflammation. Nature. 2008;454:436-44.

10. McMillan DC, Elahi MM, Sattar N, et al. Measurement of the systemic inflammatory response predicts cancer-specific and non-cancer survival in patients with cancer. Nutr Cancer. 2001;41:64-9.

11. Roxburgh CS, McMillan DC. Role of systemic inflammatory response in predicting survival in patients with primary operable cancer. Future Oncol. 2010;6:149-63.

12. Vigano A, Bruera E, Jhangri GS, et al. Clinical survival predictors in patients with advanced cancer. Arch Intern Med. 2000;160:861-8.

13. Maltoni M, Caraceni A, Brunelli C, et al. Prognostic factors in advanced cancer patients: evidence-based clinical recommendations - a study by the Steering Committee of the European Association for Palliative Care. J Clin Oncol. 2005;23:6240-8.

14. Laird BJ, Kaasa S, McMillan DC, et al. Prognostic factors in patients with advanced cancer: a comparison of clinicopathological factors and the development of an inflammation-based prognostic system. Clin Cancer Res. 2013;19:5456-64

15. Ikeya T, Shibutani M, Maeda $\mathrm{K}$, et al. Maintenance of the nutritional prognostic index predicts survival in patients with unresectable metastatic colorectal cancer. J Cancer Res Clin Oncol. 2015;141:307-13.

16. Templeton AJ, McNamara MG, Seruga B, et al. Prognostic role of neutrophil-to-lymphocyte ratio in solid tumors: a systematic review and meta-analysis. J Natl Cancer Inst. 2014;106:dju124.

17. Kim EY, Kim YS, Seo JY, et al. The Relationship between Sarcopenia and Systemic Inflammatory Response for Cancer Cachexia in Small Cell Lung Cancer. PLoS One. 2016;11:e0161125.

18. Kasmann L, Bolm L, Schild SE, et al. Neutrophil-to-Lymphocyte Ratio Predicts Outcome in Limited Disease Small-cell Lung Cancer. Lung. 2017;195:217-24.

19. Zhou $\mathrm{T}$, Hong $\mathrm{S}, \mathrm{Hu} \mathrm{Z}$, et al. A systemic inflammation-based prognostic scores (mGPS) predicts overall survival of patients with small-cell lung cancer. Tumour Biol. 2015;36:337-43.

20. Zhou T, Zhan J, Hong S, et al. Ratio of C-Reactive Protein/Albumin is An Inflammatory Prognostic Score for Predicting Overall Survival of Patients with Small-cell Lung Cancer. Sci Rep. 2015;5:10481.

21. Hong S, Zhou T, Fang W, et al. The prognostic nutritional index (PNI) predicts overall survival of small-cell lung cancer patients. Tumour Biol. 2015;36:3389-97.

22. Forrest LM, McMillan DC, McArdle CS, et al. Evaluation of cumulative prognostic scores based on the systemic inflammatory response in patients with inoperable non-small-cell lung cancer. Br J Cancer. 2003;89:1028-30.

23. Kinoshita A, Onoda $\mathrm{H}$, Imai N, et al. The $\mathrm{C}$-reactive protein/albumin ratio, a novel inflammation-based prognostic score, predicts outcomes in patients with hepatocellular carcinoma. Ann Surg Oncol. 2015;22:803-10.
24. Shibutani $\mathrm{M}$, Maeda $\mathrm{K}$, Nagahara $\mathrm{H}$, et al. The pretreatment albumin to globulin ratio predicts chemotherapeutic outcomes in patients with unresectable metastatic colorectal cancer. BMC Cancer. 2015;15:347.

25. Landskron $G$, De la Fuente $M$, Thuwajit $P$, et al. Chronic inflammation and cytokines in the tumor microenvironment. J Immunol Res. 2014;2014:149185.

26. Jafri SH, Shi R, Mills G. Advance lung cancer inflammation index (ALI) at diagnosis is a prognostic marker in patients with metastatic non-small cell lung cancer (NSCLC):a retrospective review. BMC Cancer. 2013;13:158.

27. Budczies J, Klauschen F, Sinn BV, et al. Cutoff Finder: a comprehensive and straightforward Web application enabling rapid biomarker cutoff optimization. PLoS One. 2012;7:e51862.

28. Balkwill F, Mantovani A. Inflammation and cancer: back to Virchow? Lancet. 2001;357:539-45.

29. Balkwill F, Charles KA, Mantovani A. Smoldering and polarized inflammation in the initiation and promotion of malignant disease. Cancer Cell. 2005;7:211-7.

30. Bohnhorst J, Rasmussen T, Moen SH, et al. Toll-like receptors mediate proliferation and survival of multiple myeloma cells. Leukemia. 2006:20:1138-44.

31. Kishiki T, Masaki T, Matsuoka H, et al. Modified Glasgow prognostic score in patients with incurable stage IV colorectal cancer. Am J Surg. 2013;206:234-40.

32. Yao $Y, Z$ hao $M$, Yuan D, et al. Elevated pretreatment serum globulin albumin ratio predicts poor prognosis for advanced non-small cell lung cancer patients. J Thorac Dis. 2014;6:1261-70.

33. Migita K, Takayama T, Saeki K, et al. The prognostic nutritional index predicts long-term outcomes of gastric cancer patients independent of tumor stage. Ann Surg Oncol. 2013;20:2647-54

34. Oremek GM, Sauer-Eppel H, Bruzdziak TH. Value of tumour and inflammatory markers in lung cancer. Anticancer Res. 2007;27:1911-5.

35. Hong S, Kang YA, Cho BC, et al. Elevated serum C-reactive protein as a prognostic marker in small cell lung cancer. Yonsei Med J. 2012;53:111-7.

36. Laky B, Janda M, Bauer J, et al. Malnutrition among gynaecological cancer patients. Eur J Clin Nutr. 2007;61:642-6.

37. Arrieta O, Michel Ortega RM, et al. Association of nutritional status and serum albumin levels with development of toxicity in patients with advanced non-small cell lung cancer treated with paclitaxel-cisplatin chemotherapy: a prospective study. BMC Cancer. 2010;10:50.

38. Espinosa E, Feliu J, Zamora P, et al. Serum albumin and other prognostic factors related to response and survival in patients with advanced non-small cell lung cancer. Lung Cancer. 1995;12:67-76.

39. Zhou B, Liu J, Wang ZM, et al. C-reactive protein, interleukin 6 and lung cancer risk: a meta-analysis. PLoS One. 2012;7:e43075

40. Chaturvedi AK, Caporaso NE, Katki HA, et al. C-reactive protein and risk of lung cancer. J Clin Oncol. 2010;28:2719-26.

41. Leitch EF, Chakrabarti M, Crozier JE, et al. Comparison of the prognostic value of selected markers of the systemic inflammatory response in patients with colorectal cancer. Br J Cancer. 2007;97:1266-70.

42. Biswas B, Rastogi S, Khan SA, et al. Hypoalbuminaemia is an independent predictor of poor outcome in metastatic Ewing's sarcoma family of tumours: a single institutional experience of 150 cases treated with uniform chemotherapy protocol. Clin Oncol (R Coll Radiol). 2014;26:722-9.

43. Jomrich G, Paireder M, Gleiss A, et al. Comparison of Inflammation-Based Prognostic Scores in a Cohort of Patients with Resectable Esophageal Cancer. Gastroenterol Res Pract. 2017;2017:1678584.

44. Temel JS, Greer JA, Muzikansky A, et al. Early palliative care for patients with metastatic non-small-cell lung cancer. N Engl J Med. 2010;363:733-42. 